\title{
Tratamento videolaparoscópico do cisto hepático
}

\section{Laparoscopic treatment of liver cysts}

Orlando Jorge Martins Torres, tCbC-Ma 1; Aline Maria Santos Farias²; Maria Helena Almeida Costa ${ }^{2}$; Mauricio Miranda Matias ${ }^{2}$; Poliana Cristina Oliveira Moreira ${ }^{3}$; Glaucia Mesquita Cordeiro ${ }^{4}$

\section{R E S U M O}

\begin{abstract}
Objetivo: Os autores apresentam a experiência no tratamento laparoscópico do cisto hepático não parasitário. Métodos: No período de maio de 2003 a agosto de 2006, treze pacientes com cisto hepático não parasitário foram submetidos a tratamento com fenestração laparoscópica. Havia dois pacientes do sexo masculino $(15,4 \%)$ e onze do sexo feminino $(84,6 \%)$, com idade média no momento do diagnóstico de 48,3 anos (variação de 35 a 72 anos). A maioria dos pacientes apresentava dor e desconforto abdominal, dispepsia e saciedade precoce ao diagnóstico. A tomografia computdorizada do abdome foi realizada em todos os pacientes e o cisto solitário diagnosticado em onze pacientes $(84,6 \%)$ e doença policística em dois pacientes $(15,4 \%)$. O tamanho médio do cisto solitário era 11,3 cm (variação de 9,5 a $17 \mathrm{~cm}$ ) e doença policística era 10,6 cm (um paciente com o maior cisto de 9,2 cm e o outro com 12,1 $\mathrm{cm}$ ). A cirurgia foi indicada devido a todos os pacientes se apresentarem sintomáticos e a técnica utilizada foi a fenestração laparoscópica. Resultados: O procedimento de fenestração laparoscópica foi realizado com êxito em todos os pacientes. 0 tempo operatório médio foi de 85 min (variação de 53 a 110 minutos). Não ocorreu óbito ou complicações intra-operatórias. Complicações pós-operatórias foram observadas em dois pacientes (15,4\%). Fístula biliar em um paciente e ascite em outro paciente, sendo tratados de forma conservadora com sucesso. O tempo médio de internação hospitalar foi de 3,5 dias (variação de 2 a 9 dias) e o seguimento médio foi de 36 meses. Foi observada uma recorrência assintomática (7,6\%) em paciente com doença policística após dois anos de seguimento. Conclusão: A fenestração laparoscópica é o método preferido para o tratamento do cisto hepático não parasitário. Este procedimento apresenta baixa morbidade associada a recorrência incomum. A adequada seleção dos pacientes e a técnica cirúrgica laparoscópica são fatores fundamentais para o tratamento destes pacientes.
\end{abstract}

Descritores: Fígado. Cistos. Hepatopatias. Laparoscopia.

\section{INTRODUÇÃO}

$\mathrm{O}_{4}^{\mathrm{s}}$ cistos hepáticos apresentam uma prevalência de $4-7 \%$, com um pequeno aumento com a idade. A distinção entre cistos verdadeiros congênitos e adquiridos é uma classificação prática. Entre os primeiros temos os cistos simples e a doença hepática policística. Os cistos hepáticos não parasitários geralmente são assintomáticos, sendo que o tamanho do cisto (maior que 5 centímetros) e o crescimento rápido, podem causar compressão e seus sintomas (dor severa, náusea, vômito, icterícia) justificando o tratamento cirúrgico, onde os avanços no acesso videolaparoscópico tem proporcionado grande aceitação. Este procedimento foi primeiro realizado por videolaparoscopia em 1991 e atualmente é considerado o tratamento de escolha para muitos pacientes com cistos hepáticos não parasitários. Tem sido particularmente indicado o destelhamento para grandes cistos solitários, ou um número limitado de cistos localizados nos segmentos anterolateral do fígado ${ }^{1-6}$. O objetivo do presente estudo é apresentar os resultados iniciais do tratamento dos cistos hepáticos por videolaparoscopia.

\section{MÉTODOS}

No período de Maio de 2003 a Agosto de 2006, 13 pacientes com cisto hepático não parasitário foram tratados por acesso videolaparoscópico. Havia onze pacientes do sexo feminino $(84,6 \%)$ e dois do sexo masculino $(15,4 \%)$, com idade variando de 35 a 72 anos (média de 48,3 anos). Todos os pacientes estavam sintomáticos no momento do diagnóstico e as principais queixas clínicas dos pacientes foram dor no abdome superior, desconforto abdominal, dispepsia e saciedade precoce. Nenhum paciente apresentou icterícia e as enzimas hepáticas estavam normais. Ao exame físico foi observado aumento do volume abdominal, particularmente nos pacientes com doença policística. Estes se apresentavam muito sintomáticos no momento do diagnóstico, com dor e desconforto abdominal. A ultra-sonografia do abdome superior foi realizada previamente em quatro pacientes (30,7\%). Todos os pacientes foram submetidos à tomografia computadorizada (TC) do abdome, confirmando o diagnóstico (Figuras 1 e 2). A TC avaliou as características do cisto, dimensão, localização, presença de doença policística, bem como doenças

Trabalho realizado na Disciplina de Cirúrgica III - Universidade Federal do Maranhão-UFMA - São Luis - MA-BR.

1. Professor Livre-Docente do Departamento de Cirurgia da Universidade Federal do Maranhão - UFMA- São Luis - MA-BR. 2. Estudante de Medicina - UFMA - São Luis - MA-BR. 3. Residente de Clínica Médica - UFMA- São Luis - MA-BR. 4. Residente de Cirurgia Geral - UFMA- São Luis - MA-BR 


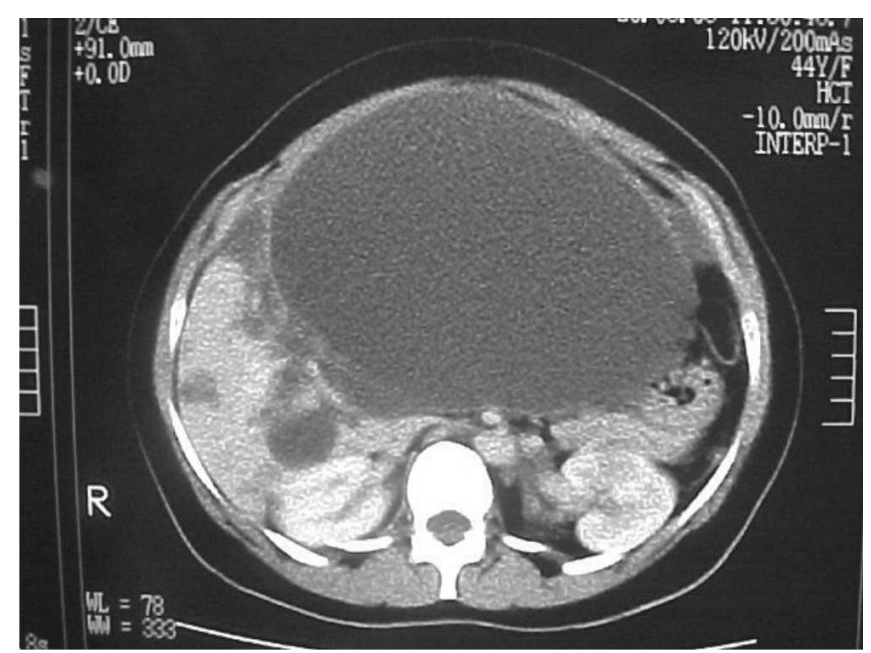

Figura 1 - Tomografia computadorizada de abdome mostrando cisto simples.

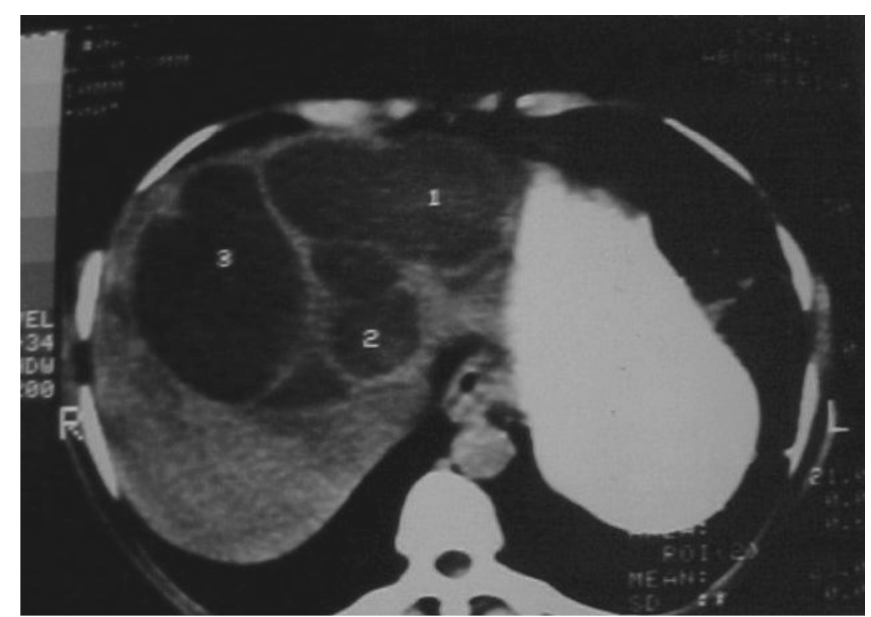

Figura 2 - $\quad$ Tomografia computadorizada de abdome mostrando doença policística do fígado.

associadas. Cisto simples solitário foi diagnosticado em onze pacientes $(84,6 \%)$ e doença hepática policística em dois pacientes $(15,4 \%)$. Os cistos simples apresentavam localização predominante no lobo direito do fígado (oito pacientes $-72,7 \%$ ). Outros cistos menores também foram observados em alguns pacientes com cistos simples. Por se tratar de cistos grandes, a localização do cisto não foi contra-indicação para a intervenção laparoscópica. O tamanho médio dos cistos simples foi de $11,3 \mathrm{~cm}$ (variando de 9,5 a $17 \mathrm{~cm}$ ). Na doença policística o tamanho médio do maior cisto foi $10,6 \mathrm{~cm}$ (um paciente com 9,2 cm e outro com 12,1 cm).

Os testes de função hepática foram realizados em todos os pacientes e se apresentavam normais. $\mathrm{Na}$ investigação diagnóstica, particular atenção foi dada para excluir cistos de natureza parasitária ou neoplásica (cistoadenoma), não tendo sido observado septações intracisto, calcificações ou projeções papilares. A indicação cirúrgica foi principalmente devido aos sintomas apresentados pelos pacientes e os exames laboratoriais pré-operatórios de rotina foram realizados em todos os pacientes. Foi utilizada a técnica de destelhamento por videolaparoscópica, nos moldes da operação preconizada por Lin no acesso convencional' 7 . O antibiótico utilizado para a profilaxia foi a cefazolina ( $2 \mathrm{~g}$ endovenoso na indução anestésica).

A abordagem laparoscópica para os cistos localizados no lobo direito ou com predominância à direita, o acesso foi o mesmo utilizado para a colecistectomia laparoscópica. Quando o cisto, ou o maior cisto, estava localizado à esquerda, foi adotado o acesso invertido da colecistectomia videolaparoscópica, sempre com a óptica através do umbigo. Nestes casos o cirurgião se posicionava à direita do paciente. Dois ou três outros portais foram utilizados, sendo um de $10 \mathrm{~mm}$ e um (ou dois) de $5 \mathrm{~mm}$.

Após ser localizado, o cisto foi puncionado utilizando uma agulha laparoscópica. Foi aspirado o maior volume de fluido possível evitando extravasamento intraperitoneal, o fluido foi enviado para estudo anatomopatológico e microbiológico. O destelhamento foi realizado utilizando um bisturi monopolar, iniciando na parte mais alta do cisto. A parede do cisto foi excisada o mais próximo possível do parênquima hepático e enviado para estudo anatomopatológico. A cavidade do cisto foi completamente examinada para identificar qualquer irregularidade que indicasse alterações neoplásicas, bem como identificar fístula biliar e sangramentos. Foi realizada drenagem da cavidade residual do cisto exteriorizando por contra-abertura abdominal em seis pacientes $(46,1 \%)$.

\section{RESULTADOS}

O procedimento foi concluído por videolaparoscopia em todos os casos, através do destelhamento do cisto, com retirada da parede deste que foi encaminhado para estudo anatomopatológico. Cistos recobertos com parênquima hepático fino também foram ressecados utilizando bisturi elétrico. Não houve complicações intra-operatórias e não houve mortalidade. O tempo operatório médio foi de 85 minutos (variando de 53 a 110 minutos). Houve a necessidade de uso de antibioticoterapia (ciprofloxacina e metronidazol), por cinco dias, em uma paciente idosa com doença policística $(7,7 \%)$, por esta apresentar em um dos cistos as características macroscópicas de secreção purulenta. Não foram observados complicações intra-operatórias nos pacientes, nem sangramento significativo, não havendo a necessidade de transfusão de concentrado de hemácias. No período pós-operatório, foram observadas complicações em dois pacientes (15,4\%). Um paciente apresentou secreção biliar através do dreno, no primeiro dia do período pós-operatório, que regrediu com medidas clínicas em seis dias, ficando internado por nove dias. Um outro paciente apresentou líquido ascítico através do dreno, sendo tratado clinicamente, sem necessidade de intervenção cirúrgica. O tempo médio de internação foi de 3,5 dias (variando de dois a nove dias). Os pacientes foram acompanhados em regime ambulatorial por até 36 meses e todos permaneceram assintomáticos durante o primeiro ano de seguimento. Recorrência foi observada através de TC em uma paciente submetida ao tra- 
tamento videolaparoscópico (7,6\%), no segundo ano de seguimento. No momento está assintomática, porém deve ser submetida a novo procedimento por videolaparoscopia. O exame anatomopatológico dos cistos não evidenciou sinais de malignidade. Não houve crescimento bacteriano no estudo microbiológico realizado no conteudo líquido do cisto. Na doença policística apenas os maiores cistos foram fenestrados e aspirados. O seguimento pós-operatório através de tomografia computadorizada foi realizado em todos os pacientes após o primeiro ano do ato operatório sem recidiva e em dez pacientes com dois anos de tratamento cirúrgico.

\section{DISCUSSÃO}

Tem sido observado um aumento da frequência em achados incidentais por exames de rotina. Embora diferentes classificações tenham sido estabelecidas, a simples distinção entre cistos congênitos verdadeiros e cistos adquiridos é bem prático. No primeiro grupo incluem o cisto simples e a doença hepática policística. Entre os cistos adquiridos são considerados os de origem tumoral (cistoadenoma e cistoadenocarcinoma), pós-traumático e o cisto hidático ${ }^{2-4}$

Cistos sintomáticos são encontrados mais comumente em mulheres com idade acima de 50 anos. Estes sintomas são relacionados ao tamanho e à localização do cisto, ou são resultado de compressão de estruturas adjacentes. Embora seja fácil atribuir os sintomas do paciente à presença de um grande cisto, a possibilidade de doença coexistente deve ser afastada antes da intervenção. Colecistopatias, doenças pépticas, infecções e tumores devem ser descartados antes dos sintomas serem atribuidos ao cisto ${ }^{3,8-11}$

Geralmente os cistos hepáticos apresentam sintomas vagos que consistem em fadiga, inapetência, náusea, febre, dispepsia, dor ou massa no abdome. Vômitos, desconforto abdominal e obstrução venosa cava podem ser observados. Da mesma forma a ruptura de um cisto com sangramento, infecção ou icterícia obstrutiva, quando presentes, apresentam indicação cirúrgica de urgência. Outras situações são quando o diagnóstico não é claro e a presença de malignidade não pode ser descartada. Nestes casos a exploração cirúrgica está indicada. Ao exame físico, hepatomegalia e massa palpável são os achados mais comuns. A icterícia pode estar evidente devido à compressão biliar. A investigação radiológica para diferenciar a lesão cística inclui inicialmente a ultra-sonografia e tomografia computadorizada de abdome. A ultra-sonografia é um procedimento não invasivo, facilmente disponível, sensível e de baixo custo, devendo ser o exame de escolha inicial. É útil para definir o número e localização dos cistos, bem como sua estrutura interna, apresentando uma especificidade de até $90 \%$. A tomografia computadorizada de abdome confirma informações diagnósticas equivalentes. Entretanto proporciona informações mais detalhadas sobre a localização e a profundidade do cisto no parênquima hepático, sendo essencial no planejamento cirúrgico, em particular quando a abordagem laparoscópica é utilizada. A ressonância nuclear magnética é menos disponível e geralmente não proporciona mais informações que a tomografia ${ }^{12,13}$. No presente estudo, todos os recursos de diagnóstico procuraram afastar a presença de doença maligna.

Os cistos congênitos, em sua maioria (90-95\%) são pequenos e assintomáticos, portanto não necesitando de qualquer tipo de terapia. Em geral estes cistos são menores que $10 \mathrm{~cm}$ de diâmetro e devem ser avaliados regularmente por ultra-sonografia. O crescimento dos cistos pode torná-los sintomáticos necessitando de tratamento ${ }^{10,11,14}$. Observamos sintomatologia clínica em todos os pacientes, sendo esta a indicação de tratamento cirúrgico.

Entre as opções de tratamento que podem ser aplicadas aos cistos hepáticos incluem a simples aspiração do cisto, escleroterapia percutânea, fenestração do cisto por laparotomia, destelhamento por via videolaparoscópica, enucleação, ressecção hepática, cistojejunostomia e transplante de fígado. A simples aspiração do cisto tem sido abandonada por resultar em recorrência em 100\% dos casos em dois anos. A aspiração combinada com escleroterapia com álcool ou outras substâncias parece ser uma alternativa para pacientes sintomáticos selecionados, inaptos para cirurgia. Pode ser observado colangite esclerosante com o uso de etanol7,8,11,15-16

O objetivo do tratamento cirúrgico é a descompressão do cisto e a prevenção da recorrência. A natureza e origem do cisto hepático são importantes fatores determinantes na abordagem terapêutica. A fenestração ampla dos cistos simples foi introduzida nos anos 60 por Lin $^{7}$ e desde então permanece como o tratamento de escoIha para os cistos simples não complicados do fígado. O princípio é o destelhamento do cisto para permitir drenagem livre na cavidade peritoneal. Como a natureza do fluido intracisto é similar aquela do soro, o fluido será reabsorvido. Uma vez que os cistos que necessitam terapia são quase sempre grandes e, portanto se tornam parte da superficie do fígado, a fenestração geralmente é um procedimento fácil. Como não há tecido hepático a ser ressecado, o destelhamento poupa tecido hepático normal. A omentoplastia adicional e a sutura das margens do cisto parecem reduzir as taxas de recorrência para 0 a $14 \%$. Desde 1991, quando a primeira fenestração laparoscópica de cisto hepático gigante solitário, em um paciente de 73 anos foi realizada, esta modalidade de tratamento passou a ser largamente utilizado e tem sido proposto mesmo para doença policística hepática. Apesar de estudos randomizados não controlados com resultados em longo prazo não serem disponíveis, o tratamento videolaparoscópico do cisto hepático não complicado é considerado o tratamento padrão. A via laparoscópica pode não ser factível em alguns pacientes devido ao acesso limitado de cistos localizados centralmente ou na região posterior dos segmentos VI, VII, VIII e IVa ${ }^{7,8,10}$. Gamblin et al, indicaram tratamento laparoscópico de cisto hepático em $92 \%$ dos pacientes devido a dor. 0 tratamento foi realizado por videolaparoscopia em 58\% 
dos pacientes, sendo que o diagnóstico pré-operatório foi de doença policística em $88 \%$ dos casos e cisto simples em $12 \%$, diagnosticados por exames de imagem ${ }^{17}$. Neste estudo foi possível a realização da fenestração laparoscópica em todos os pacientes, sem a necessidade de conversão.

A recorrência das manifestações da doença cística, causada pela regeneração dos cistos hepáticos após o tratamento por videolaparoscopia, tem sido o principal obstáculo à aceitação maior do destelhamento videolaparoscópico do cisto congênito. Esta situação foi observada em até metade dos pacientes após a operação. A fenestração laparoscópica reduz a pressão intra-abdominal, causando regeneração do cisto até que a pressão intraabdominal se eleve novamente. Entretanto recentes trabaIhos têm registrado taxa total de recorrência de somente 4 a $10,5 \%$ em pacientes com um limitado número de cistos congênitos. Em casos de cistos solitários não têm sido registrada recorrência mesmo durante seguimento em longo prazo. Isto tem sido atribuido ao aumento da experiência com a técnica laparoscópica, pois as recorrências diagnosticadas estão geralmente relacionadas aos pacientes com destelhamento incompleto do cisto. Nestes casos pode ser observado até $40 \%$ de recorrência. Alguns detaIhes técnicos para reduzir as taxas de recorrência são a seleção criteriosa dos pacientes, excluindo aqueles pacientes com sintomas não relacionados ao cisto, ressecção ampla da parede do cisto, hemostasia cuidadosa da superfície do cisto, cauterização da cavidade cística e a utilização de omento na cavidade cística ${ }^{10,17}$.

Alguns cirurgiões continuam relutantes a realizar ressecções maiores devido ao risco de fístula biliar de ductos de dentro do septo do cisto ou hemorragia do parênquima hepático ressecado, que pode ser difícil de ser manuseado laparoscopicamente. A fenestração laparoscópica do cisto hepático, no presente estudo, foi realizada com mínima morbidade e, na maioria dos casos, necessitou de dois dias de internação hospitalar. As complicações observadas na literatura resultante do tratamento videolaparoscópico incluem ascite, derrame pleural, íleo prolongado, sangramento. As causas mais comuns de conversão são cistos profundos dentro do parênquima hepático, cistos localizados nos segmentos VI, VII, VIII e IVa, sangramento não controlado ou uma lesão maior da via biliar. A ressecção aberta resulta em melhora dos sintomas em longo prazo, semelhante ao procedimento videolaparoscópico, entretanto apresenta um aumento da morbidade perioperatória (50 a $58 \%)^{8,10,17-20}$.

A doença hepática policística é uma entidade clínica rara com uma prevalência de 0,05 a 0,13 em estudos de autópsia. Está mais comumente associada com doença renal policística autossômica dominante, onde o desenvolvimento de cistos hepáticos ocorre após o início dos cistos renais. A incidência de cistos hepáticos na doença renal policística varia de 29 a $48 \%$ dependendo da população examinada e do método diagnóstico usado. A frequência de cistos hepáticos em pacientes com doença renal aumenta com a idade da população em estudo, com uma prevalência de 11 a 27\% em pacientes com idade inferior a 30 anos e 77 a $83 \%$ em pacientes acima de 60 anos. Outras causas genéticas tem sido associadas que não os cistos renais. A maioria dos casos de doença hepática policística tem comportamento assintomático, porém pode causar dor abdominal, distensão abdominal ou saciedade precoce devido a hepatomegalia. Mais raramente complicações do cisto podem incluir a infecção, hemorragia, ruptura com hemoperitôneo, torção, hipertensão porta ou icterícia devido à compressão do ducto biliar 2,3,8,15,16,21.

Os resultados da fenestração laparoscópica em pacientes com doença hepática policística não são semeIhantes aos do cisto simples. Gigot et a ${ }^{18}$, classificam como tipo 1 (menos de 10 cistos maiores que $10 \mathrm{~cm}$ cada), tipo 2 (múltiplos cistos de tamanho médio com algum parênquima hepático entre os cistos), tipo 3 (lesões tipo 2 sem parênquima entre os cistos). O tipo 1 é o mais adequado para o tratamento laparoscópico. Recorrência ocorre com maior frequência em doença policística quando comparado com cisto solitário (61\% versus $23 \%$ ). Segundo a classificação de Morino et al. ${ }^{20}$, na doença tipo I em que um limitado número de cistos grandes estão localizados no segmento anterior do fígado, a fenestração laparoscópica está indicada. No tipo Il, em que um grande número de pequenos cistos está localizado por todo o fígado, o procedimento está contra-indicado. Apesar do papel da fenestração laparoscópica no manuseio da doença policística permanecer indefinido, este e outros resultados sugerem que a fenestração laparoscópica oferece benefício para um grupo selecionado de pacientes, particularmente aqueles que não são adequados à punção percutânea, aspiração ou esclerose. Nestes casos a fenestração laparoscópica do cisto hepático deveria ser considerada uma opção de primeira linha no tratamento cirúrgico de pacientes sintomáticos antes de ser considerada para fenestração aberta, ressecção hepática ou transplante hepático. No paciente com doença renal policística avançada ou em diálise, pode ser observado um aumento das taxas de complicações associadas à abordagem laparoscópica pelo risco de sangramento e infecção. Especificamente na doença policística hepática a fenestração laparoscópica é segura, resulta em alívio dos sintomas relacionados à doença e a repetição do procedimento pode ser necessário em até 30\% dos pacientes 17,19 22.

A fenestração laparoscópica é o método de escolha para o tratamento do cisto hepático solitário não parasitário, em paciente sintomático. É um procedimento efetivo, com baixa morbidade, sendo incomum a recorrência. O papel da laparoscopia na doença policística do fígado, tem demonstrado resultados animadores. A experiência da equipe em cirurgia videolaparoscópica bem como a escoIha adequada do paciente são fatores fundamentais para o resultado do tratamento. 


\section{A B S T R A C T}

Objective: The authors present their experience with the laparoscopic management of non-parasitic liver cysts. Methods: During the period from May 2003 to August 2006, thirteen patients with non-parasitic liver cysts underwent laparoscopic fenestration. Eleven patients were female (84.6\%) and two (15.4\%) were male. The average age at diagnosis was 48.3 years (range 35-72). The majority of patients presented with pain, discomfort, dyspepsia, or early satiety at diagnosis. Computed tomography scans were performed in all cases. The cysts were solitary in eleven patients (84.6\%) and polycystic liver disease in two patients (15.4\%). The average size of the solitary cysts was $11.3 \mathrm{~cm}$ (range $9.5-17 \mathrm{~cm}$ ), and the polycystic liver disease was $10.6 \mathrm{~cm}($ range $9.2-12.1 \mathrm{~cm})$. Operation was indicated due to symptoms in all patients. The technique performed was laparoscopic cyst fenestration. Results: Laparoscopic fenestration was completed in all patients. Mean operative time was 85 minutes (range 53-110). Intraoperative complications were not detected and there was no death. Postoperative complications were observed in two patients (15.4\%). Bile leakage was present in one patient, and ascites in another patient. Both cases were treated conservatively. The average hospital stay was 3.5 days (range 2-9 days). Mean follow-up was 36 months. There was one asymptomatic recurrence (7.6\%) in a patient with polycystic liver disease after two years of follow-up. Conclusion: Laparoscopic fenestration is the preferred method of treatment for non-parasitic liver cyst. This operation causes low morbidity and the recurrence is uncommon. Adequate selection of patients and the laparoscopic technique are important for the treatment.

Key words: Liver. Cysts. Hepatopathy. Laparoscopic.

\section{REFERÊNCIAS}

1. Szabó LS, Takács I, Arkosy P, Sápy P, Szentkereszty Z. Laparoscopic treatment of nonparasitic hepatic cysts. Surg Endosc. 2006; 20(4):595-7. Epub 2006 Jan 25.

2. Bistritz L, Tamboli C, Bigam D, Bain VG. Polycystic liver disease: experience at a teaching hospital. Am J Gastroenterol. 2005; 100(10):2212-7

3. Robinson TN, Stiegmann GV, Everson GT. Laparoscopic palliation of polycystic liver disease. Surg Endosc. 2005; 19(1):130-132. Epub 2004 Nov 11.

4. Weber T, Sendt W, Scheele J. Laparoscopic unroofing of nonparasitic liver cysts within segments VII and VIII: technical considerations. J Laparoendosc Adv Surg Tech. 2004; 14(1):37-42.

5. Paterson-Brown S, Garden OJ. Laser-assisted laparoscopic excision of liver cyst. Br J Surg. 1991; 78(9):1047-8.

6. Fabiani P, Katkhouda N, lovine L, Mouiel J. Laparoscopic fenestration of biliary cysts. Surg Laparosc Endosc. 1991; 1(3):162-5.

7. Lin TY, Chen CC, Wang SM. Treatment of non-parasitic cystic disease of the liver: a new approach to therapy with polycystic liver disease. Ann Surg. 1968; 168(5):921-7

8. Giuliante F, D'Acapito F, Vellone M, Giovannini I, Nuzzo G. Risks for laparoscopic fenestration of liver cysts. Surg Endosc. 2003; 17(11):1735-8. Epub 2003 Jun 17.

9. Fiamingo P, Tedeschi U, Veroux M, Cillo U, Brolese A, Rold A et al. Laparoscopic treatment of simple hepatic cysts and polycystic liver disease. Surg Endosc. 2003; 17(4):623-6. Epub 2003 Feb 10.

10. Gloor B, Ly Q, Candinas D. Role of laparoscopy in hepatic cyst surgery. Dig Surg. 2002; 19(6):494-9.

11. Petri A, Höhn J, Makula E, Kókai EL, Savanya GK, Boros M, Balogh A. Experience with different methods of treatment of nonparasitic liver cysts. Langenbeck's Arch Surg. 2002; 387(5-6):229-33. Epub 2002 Sep 6.

12. Tocchi A, Mazzoni G, Costa G, Cassani D, Bettelii E, Agostini N, Miccini M. Symptomatic nonparasitic hepatic cysts: options for and results of surgical management. Arch Surg. 2002; 137(2):154-8.

13. Ammori BJ, Jenkins BL, Lim PC, Prasad KR, Pollard SG, Lodge PJ. Surgical strategy for cystic diseases of the liver in a Western hepatobiliary center. World J Surg. 2002; 26(4):462-9. Epub 2002 Feb 6.

14. Cellier C, Cuenod CA, Deslandes P, Auroux J, Landi B, Siauve N, Barbier JP, Frija G. Symptomatic hepatic cysts: treatment with single-shot injection of minocycline hydrochloride. Radiology. 1998; 206(1):205-9.

15. Que F, Nagorney DM, Gross JB Jr, Torres VE. Liver resection and cyst fenestration in the treatment of severe polycystic liver disease. Gastroenterology. 1995; 108(2):487-94.

16. Swenson K, Seu P, Kinkhabwala M, Maggard M, Martin P, Gross J, Busuttil R. Liver transplantation for adult polycystic Iver disease. Hepatology. 1998; 28(2):412-5.

17. Gamblin TC, Holloway SE, Heckman JT, Geller DA. Laparoscopic resection of benign hepatic cysts: a new standard. J Am Coll Surg. 2008; 207(5):731-6. Epub 2008 Aug 30.

18. Gigot JF, Legrand M, Hubens G, de Canniere L, Wibin E, Deweer F et al. Laparoscopic treatment of nonparasitic liver cysts: adequate selection of patients and surgical technique. World J Surg. 1996; 20(5):556-61.

19. Morino M, De Giuli M, Festa V, Garrone C. Laparoscopic management of symptomatic nonparasitic cysts of the liver. Indications and results. Ann Surg. 1994; 219(2):157-64.

20. Koperna T, Vogl S, Satzinger U, Schulz F. Nonparasitic cysts of the liver: results and options of surgical treatment. World I Surg. 1997; 21(8):850-4; discussion 854-5.

21. Gigot JF, Jadoul P, Que F, Van Beers BE, Etienne J, Horsmans $Y$ et al. Adult polycystic liver disease: is fenestration the most adequate for long-term management? Ann Surg. 1997; 225(3):286-94.

22. Mekeel KL, Moss AA, Reddy KS, Mulligan DC, Harold KL. Laparoscopic fenestration of giant hepatic cysts. Surg Laparosc Endosc Percutan Tech. 2008; 18(5):511-3.

Recebido em 09/12/2008

Aceito para publicação em 18/02/2009

Conflito de interesse: nenhum

Fonte de financiamento: nenhuma

\section{Como citar este artigo:}

Torres OJM, Farias AMS, Costa MHA, Matias MM, Moreira PCO, Cordeiro GM. Tratamento videolaparoscópico do cisto hepático. Rev Col Bras Cir. [periódico na Internet] 2009; 36(6). Disponível em URL: http:/ /www.scielo.br/rcbc

\section{Endereço para correspondência:}

Dr. Orlando Torres

E-mail: o.torres@uol.com.br 